\title{
Rendimento e concentração de nutrientes em alface, em função das adubações orgânica e mineral ${ }^{1}$.
}

\author{
Edson Talarico Rodrigues ${ }^{2}$; Vicente Wagner D. Casali ${ }^{3}$ \\ ${ }^{2}$ UFMS-Depto de Ciências Agrárias, C. Postal 533, 79.804-970, Dourados-MS; ${ }^{3}$ UFV-Depto de Fitotecnia, 36.570-000, Viçosa-MG. \\ * Bolsista CNPq.
}

\begin{abstract}
RESUMO
O rendimento e a concentração de nutrientes na alface, cultivar Babá, foram quantificados em função das adubações orgânica e mineral, em solo distrófico. Utilizou-se o sistema de transplante de mudas em vasos de polietileno de $5 \mathrm{dm}^{3}$, em casa de vegetação e o delineamento experimental inteiramente casualizado com três repetições. Os tratamentos foram dispostos no arranjo fatorial 4 x 3 , com quatro doses de composto orgânico $\left(0 ; 0,9 ; 1,8\right.$ e $2,7 \mathrm{dm}^{3}$, equivalentes a 0,33, 66 e 99 t/ha, na base seca) e três níveis de adubação mineral $(0,1$ e 2$)$. O nível 2 veiculou as doses de macro e micronutrientes apropriadas e o nível 1 consistiu de metade do nível 2. As produtividades máximas foram estimadas em 119,5, 119,4 e $153,9 \mathrm{~g} /$ planta, com as doses de 37,7, 18,9 e 13 t/ha de composto, nos níveis 0,1 e 2 de adubo mineral, respectivamente. As doses de composto orgânico promoveram aumentos menos acentuados que a adubação mineral nas concentrações foliares de $\mathrm{N}$, elevaram as concentrações de $\mathrm{P}, \mathrm{K}$ e $\mathrm{Na}$ e diminuíram as de $\mathrm{Ca}$, demonstrando que doses altas de adubos orgânicos provocam excesso de cátions monovalentes, prejudicando a absorção do Ca.
\end{abstract}

Palavras-Chave: Lactuca sativa L., Adubação organo-mineral, nutrição mineral.

\begin{abstract}
Yield and nutrient concentration in lettuce as a function of organic and mineral manuring.

Nutrient accumulation in lettuce plants, cv. Babá, was quantified as a function of organic and mineral fertilisers, in a low fertility soil. The experiment was established by transplanting seedlings to $5 \mathrm{dm}^{3}$ capacity pots in a greenhouse. The experiment was laid out in a complete randomised block design with three replications ordered in factorial arrangement $(4 \times 3)$ of four doses of organic compost $(0$, $0.9,1.8$ and $2.7 \mathrm{dm}^{3}$; equivalent to $0,33,66$, and $99 \mathrm{t} / \mathrm{ha}$, in drought basis) and three mineral fertiliser levels ( 0,1 and 2). Level two carried doses of macro and micronutrients normally recommended for the culture, with level one carrying half the dose of level two. The maximum estimated yields were $119.5,119.4$ and $153.9 \mathrm{~g} / \mathrm{plant}$ with 37.7, 18.9 and 13 t/ha compost doses, for levels 0,1 and 2, respectively. Organic compost doses resulted in a less intense increase in $\mathrm{N}$ foliar concentration, compared with mineral fertilizers, increased $\mathrm{P}, \mathrm{K}$, and $\mathrm{Na}$ concentrations and diminished $\mathrm{Ca}$ concentrations, revealing that organic fertilisers promote monovalent cations in high doses, prejudicing $\mathrm{Ca}$ absorption.
\end{abstract}

Keywords: Lactuca sativa L, organic-mineral fertilizers, mineral nutrition.

\section{(Aceito para publicação em 19 de abril de 1999)}

A alface, assim como várias outras hortaliças, exige um fornecimento considerável de nutrientes prontamente solúveis, dentro de um curto período de intenso crescimento vegetativo (Zink \& Yamaguchi, 1962; Garcia et al., 1982).

Por serem fontes de nutrientes e por beneficiarem propriedades físicas, químicas e biológicas do solo, os adubos orgânicos são amplamente recomendados para hortaliças como a alface (Sonnenberg, 1985; Kiehl; 1985, Comissão ..., 1989). São condicionadores eficientes do solo, reduzindo a densidade aparente, favorecendo a formação de agregados estáveis (Melo et al., 1984), aumentando a capacidade de aeração, de infiltração e de armazenamento de água (Grohmann, 1972; Alisson, 1973). Podem melhorar a disponibilidade dos nutrientes pelo aumento de $\mathrm{pH}$, pelo aumento da atividade de macro e microrganismos, pelos efeitos indiretos da melhoria de propriedades físicas, químicas e biológicas do solo (Mazur et al. 1983; Kiehl, 1985; Siqueira, 1988).

A atuação conjunta desse e de inúmeros outros efeitos resulta, em muitos casos, em produtividades de alface que podem superar as obtidas com adubos minerais (Trocmé \& Briant, 1963; Hawort \& Cleaver, 1967; Hartrath, 1986).

No cultivo da alface é comum a utilização de doses altas de adubos orgânicos e minerais para atender à demanda de nutrientes. Por isso, pesquisas que estudem a interação entre doses e fontes (orgânicas e minerais) podem eliminar desperdícios e evitar efeitos fitotóxicos, pois sabe-se que doses muito altas de adubos desbalanceiam as relações entre nutrientes e salinizam o solo (Shortall \& Liebhardt, 1975; Gianello \& Ernani, 1983; Wang et al., 1984; Valente, 1985, Rodrigues, 1990).

Este trabalho objetivou quantificar o rendimento e o acúmulo de nutrientes na alface em função de doses de composto orgânico e de adubos minerais, num solo distrófico, visando critérios específicos de adubação organo-mineral.

\section{MATERIAL E MÉTODOS}

$\mathrm{O}$ experimento foi conduzido em casa de vegetação, na Universidade $\mathrm{Fe}$ deral de Viçosa, utilizando-se amostras dos primeiros $20 \mathrm{~cm}$ de um Latossolo Vermelho Amarelo distrófico, texturalmente classificado como argila.

\footnotetext{
${ }^{1}$ Parte da dissertação de mestrado do primeiro autor, apresentada à Universidade Federal de Viçosa, Viçosa-MG.
} 
Tabela 1. Equações de regressão ajustadas para o peso da matéria fresca (PFA) e as concentrações foliares de N, P e K em função das doses de composto (C) e dos níveis de adubo mineral (M) e de Ca e Na em função das doses de composto. Viçosa, UFV, 1988.

\begin{tabular}{llc}
\hline $\begin{array}{c}\text { Variável } \\
\text { Dependente }\end{array}$ & \multicolumn{1}{c}{ Equação } & $\mathbf{R}^{2}$ \\
\hline $\mathrm{PFA}$ & $\mathrm{Y}=19,3891+32,5611 \mathrm{C}^{2}, 5-9,5323 \mathrm{CM}^{0,5}-2,6504 \mathrm{C}+50,0943 \mathrm{M}$ & $0,913^{* *}$ \\
$\mathrm{~N}$ & $\mathrm{Y}=2,1259+0,1986 \mathrm{C} 0^{5}+1,0411 \mathrm{M}^{0,5}-0,1851 \mathrm{CM}^{0,5}-0,000453 \mathrm{C}+0,4375 \mathrm{M}$ & $0,931^{* *}$ \\
$\mathrm{P}$ & $\mathrm{Y}=0,1723+0,005313 \mathrm{C}+0,1125 \mathrm{M}$ & $0,936^{* *}$ \\
$\mathrm{~K}$ & $\mathrm{Y}=4,0238+0,2693 \mathrm{C}+1,3146 \mathrm{M}-0,0861 \mathrm{CM}-0,0039 \mathrm{C}^{2}+6,27 \times 10^{-4} \mathrm{C}^{2} \mathrm{M}+1,9 \times 10^{-5} \mathrm{C} 3$ & $0,985^{* *}$ \\
$\mathrm{Ca}$ & $\mathrm{Y}=0,8210-0,003455 \mathrm{C}$ & $0,925^{* *}$ \\
$\mathrm{Na}$ & $\mathrm{Y}=0,1599+0,0041 \mathrm{C}-0,000014 \mathrm{C}^{2}$ & $0,993^{* *}$ \\
\hline
\end{tabular}

** Coeficientes de determinação significativos a $1 \%$ de probabilidade pelo teste $\mathrm{F}$.

Os resultados da análise química do solo foram: $\mathrm{pH}$ (água) 4,5; $\mathrm{Ca}+\mathrm{Mg}$ trocável $=0,1 ; \mathrm{Al}$ trocável $=0,6$, expressos em $\mathrm{cmol}{ }_{\mathrm{c}} / \mathrm{dm}^{3} ; \mathrm{P}=0,8 \mathrm{mg} / \mathrm{dm}^{3}$ (extraído pelo Mehlich 1); $\mathrm{K}=0,05 \mathrm{cmol}{ }_{c} / \mathrm{dm}^{3}$, todos determinados de acordo com a metodologia descrita em Defelipo e Ribeiro (1981) e carbono orgânico = 1,36 $\mathrm{dag} / \mathrm{kg}$ pelo método Walkley-Black (Jackson, 1958).

O composto orgânico foi produzido com a mistura de palhada de milho seca e triturada, juntamente com esterco de bovinos, de acordo com as recomendações de Loures (1983). Antes da incorporação ao solo, o composto foi passado em peneira com malha de $4 \mathrm{~mm}$, ocasião em que tinha as seguintes características: $\mathrm{pH}\left(\mathrm{H}_{2} \mathrm{O}\right)=8,7$; matéria orgânica = 35,2 $\mathrm{dag} / \mathrm{kg}$, pelo método da perda por ignição (Kiehl, 1985); teores totais, em dag/ $\mathrm{kg}$, de $\mathrm{P}=1,0 ; \mathrm{K}=2,75 ; \mathrm{Ca}=0,86 \mathrm{Mg}=$ 0,55 e $\mathrm{Na}=3,36$, determinados de acordo com Sarruge e Haag (1974).

O delineamento experimental utilizado foi inteiramente casualizado, com três repetições. A unidade experimental consistiu de um vaso de polietileno, preenchido com $4,5 \mathrm{dm}^{3}$ de substrato (mistura de solo com as doses de composto orgânico), com uma planta por vaso. Os tratamentos foram dispostos num arranjo fatorial $4 \times 3$, com quatro doses de composto orgânico $(0 ; 0,9 ; 1,8$ e $2,7 \mathrm{dm}^{3}$, equivalentes a $0 ; 33 ; 66$ e 99 t/ha, com base na matéria seca) e três níveis de adubação mineral $(0 ; 1$ e 2$)$.

O nível 2 de adubação continha os nutrientes em doses apropriadas para a nutrição das plantas, de acordo com Novais et al. (1991). Neste nível foram adicionados os seguintes nutrientes por $\mathrm{dm}^{3}$ de substrato: $150 \mathrm{mg}$ de $\mathrm{N} ; 300 \mathrm{mg}$ de P; $150 \mathrm{mg}$ de K; $40 \mathrm{mg}$ de $\mathrm{S} ; 2 \mathrm{mg}$ de B; $1 \mathrm{mg}$ de $\mathrm{Cu} ; 2 \mathrm{mg}$ de $\mathrm{Zn}$ e 0,3 mg de Mo, na forma dos sais puros $\mathrm{NH}_{4} \mathrm{H}_{2} \mathrm{PO}_{4}$, $\mathrm{KH}_{2} \mathrm{PO}_{4}, \mathrm{Ca}\left(\mathrm{H}_{2} \mathrm{PO}_{4}\right)_{2} \cdot \mathrm{H}_{2} \mathrm{O}, \mathrm{H}_{3} \mathrm{BO}_{3}$, $\mathrm{CuSO}_{4}, \mathrm{ZnSO}_{4} \mathrm{e} \mathrm{NaMoO}_{4} . \mathrm{H}_{2} \mathrm{O}$. O nível 1 consistiu da metade dos nutrientes do nível 2 e o nível 0 representou a ausência de adubação mineral. Nos níveis $1 \mathrm{e}$ 2 o $\mathrm{N}$ foi parcelado em $1 / 3$ na adubação básica e 2/3 em duas aplicações, aos 7 e 14 dias após o transplante. As adubações mineral e orgânica foram feitas 15 dias antes do transplante.

A cultivar Babá de Verão foi semeada em 13 de julho de 1988 utilizando-se bandeja de plástico contendo uma mistura composta por solo, areia grossa, composto orgânico e a dose de $200 \mathrm{~g} / \mathrm{m}^{2}$ de superfosfato simples. O transplante de mudas com raízes "nuas" foi feito aos 28 dias após a semeadura e a colheita, 36 dias após o transplante. A irrigação foi feita com água desmineralizada, diariamente.

As plantas foram colhidas pela manhã, entre seis e sete horas, para evitarem-se imprecisões na determinação do peso da matéria fresca. Após a pesagem, foram secas em estufa com ventilação forçada de ar a $60^{\circ} \mathrm{C}$. Todas as folhas foram moídas e passadas em peneira com malha de 20 mesh. Para a determinação da concentração de nutrientes nas folhas, foi feita a digestão com $\mathrm{HClO}_{4} \mathrm{e}$ $\mathrm{H}_{2} \mathrm{O}_{2}$. O K foi determinado por fotometria de chama, o $\mathrm{N}$, pelo método de Nessler; o P, por colorimetria e o Ca, por espectrofotometria de absorção atômica, de acordo com metodologias descritas em Sarruge \& Haag (1974).

Realizaram-se as análises de variância e o ajuste de equações de regressão da matéria fresca da parte aérea e da concentração de nutrientes no tecido foliar, como variáveis dependentes das doses de composto e dos níveis de adubo mineral. Foram selecionadas as equações com coeficientes de determinação maiores que 0,900 , com o quadrado médio do devido à regressão significativo a $1 \%$ e com o quadrado médio do desvio da regressão não significativo a $5 \%$ de probabilidade, pelo teste $\mathrm{F}$.

Nas variáveis em que o efeito da interação composto $\mathrm{x}$ adubo mineral foi significativo, as equações de regressão foram desmembradas para as doses de composto em cada nível de adubo mineral, pela metodologia de "cortes".

\section{RESULTADOS E DISCUSSÃO}

A produção de matéria fresca da parte aérea foi influenciada pelas doses de composto orgânico $(\mathrm{C})$, pelos níveis de adubo mineral $(\mathrm{M})$ e pela interação C x M. As estimativas das produtividades máximas, foram 119,5, 119,4 e 153,9 g/planta, obtidas com as doses de 37,7, 18,9 e 13 t/ha de composto orgânico (base seca), respectivamente, para os níveis 0,1 e 2 de adubação mineral (Tabela 1 e Figura 1).

O nível 2 de adubação mineral promoveu as maiores produtividades. Foram adicionadas doses equivalentes a 108 de N; 216 de P; 108 de K e 28,8 de S, em $\mathrm{kg} / \mathrm{ha}$, mais micronutrientes, compatíveis com as recomendações de adubação para a cultura. Nesse nível, doses maiores que 13 t/ha de composto orgânico reduziram o peso das plantas. No nível 1 e na ausência de adubo mineral (nível 0), foram 
necessárias doses maiores de composto para a obtenção das produtividades máximas. Nos três níveis de adubo mineral, doses acima da que produziu o peso máximo de plantas provocaram condições de fitotoxidez (Figura 1).

As interações verificadas entre as fontes mineral e orgânica (Figura 1) demonstram a possibilidade de estabelecer alternativas mais baratas de adubação, especialmente para os locais em que os adubos orgânicos são disponíveis a baixos custos. A estratégia consiste na utilização de fontes orgânicas em doses que maximizam a produtividade, sendo os adubos minerais adicionados em doses apenas complementares.

As doses crescentes de composto orgânico promoveram aumentos de nitrogênio menos intensos que os níveis de adubo mineral (Figura 2A), porque as fontes orgânicas fornecem o $\mathrm{N}$ inorgânico gradualmente, na medida em que se processa a mineralização da matéria orgânica. Resultados de experimentos sobre a mineralização do $\mathrm{N}$ em resíduos de origem animal no solo, estabelecem taxas entre 13 e $67 \%$ após 6 meses. Ocorreu pouca mineralização no primeiro mês, aumentos até aos 3 meses e estabilização na liberação do $\mathrm{N}$ até aos 6 meses (Chae \& Tabatabai, 1986). Resultados de Cassol et al. (1997) também demonstram essa variação. Após 220 dias de incubação, as camas de aviário estudadas mineralizaram cerca de $35 \%$ e o esterco de suínos $14 \%$ do $\mathrm{N}$ total adicionado ao solo; enquanto que o esterco de bovinos estudado provocou a imobilização do $\mathrm{N}$ do solo. Os percentuais de mineralização desses experimentos são indícios de que o $\mathrm{N}$ inorgânico liberado pelos adubos orgânicos é insuficiente para o atendimento da demanda nutricional da alface durante o curto período entre o transplante e a colheita, sendo portanto necessárias complementações com fontes minerais.

Suprimentos menores e graduais do $\mathrm{N}$ podem ser vantajosos, até limites aceitáveis de redução no peso, porque as plantas passam a fechar melhor a cabeça (Asano, 1984) e esta característica é comercialmente desejável em cultivares dos grupos repolhuda crespa e repolhuda lisa.

As doses do composto orgânico promoveram acréscimos lineares do $\mathrm{P}$ to-

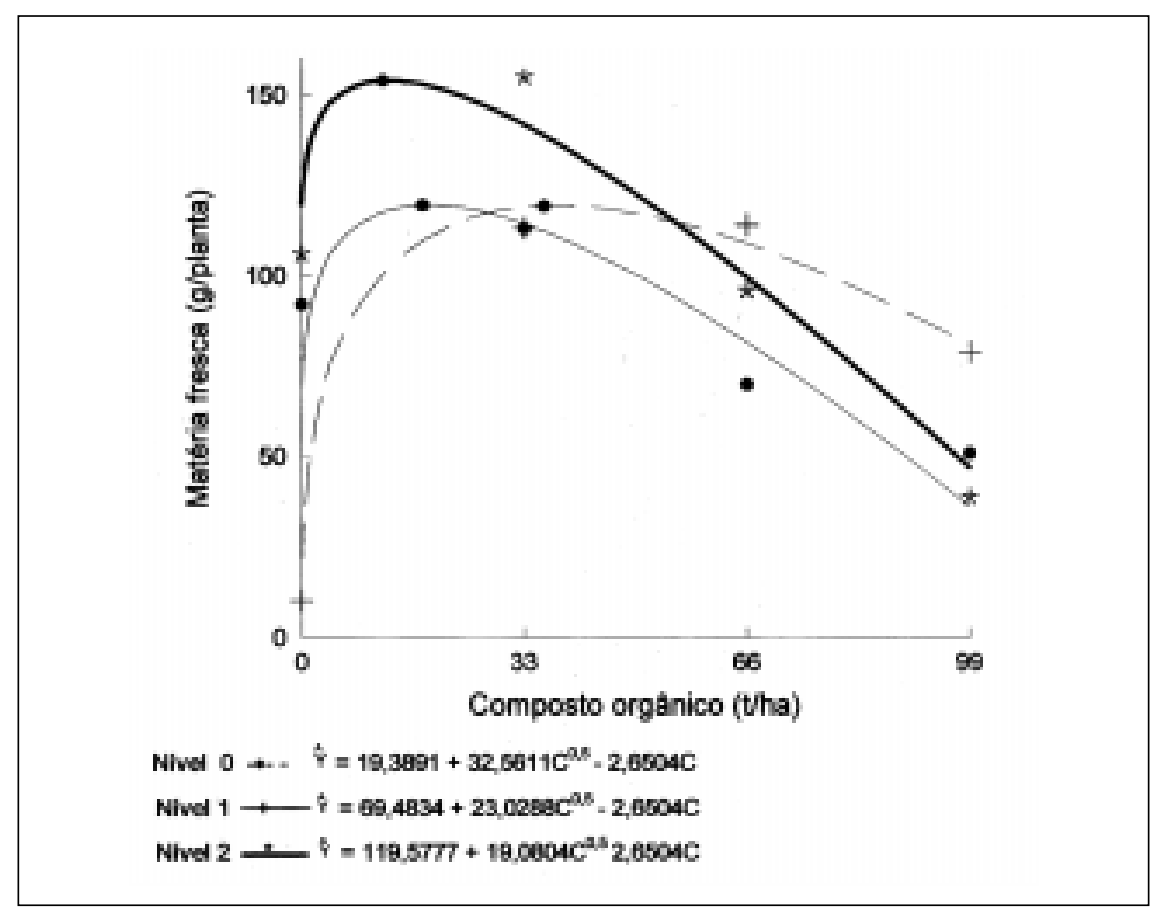

Figura 1. Produção de alface, em g/pé, em função das doses de composto orgânico e dos níveis de adubo mineral. Viçosa, UFV 1988.

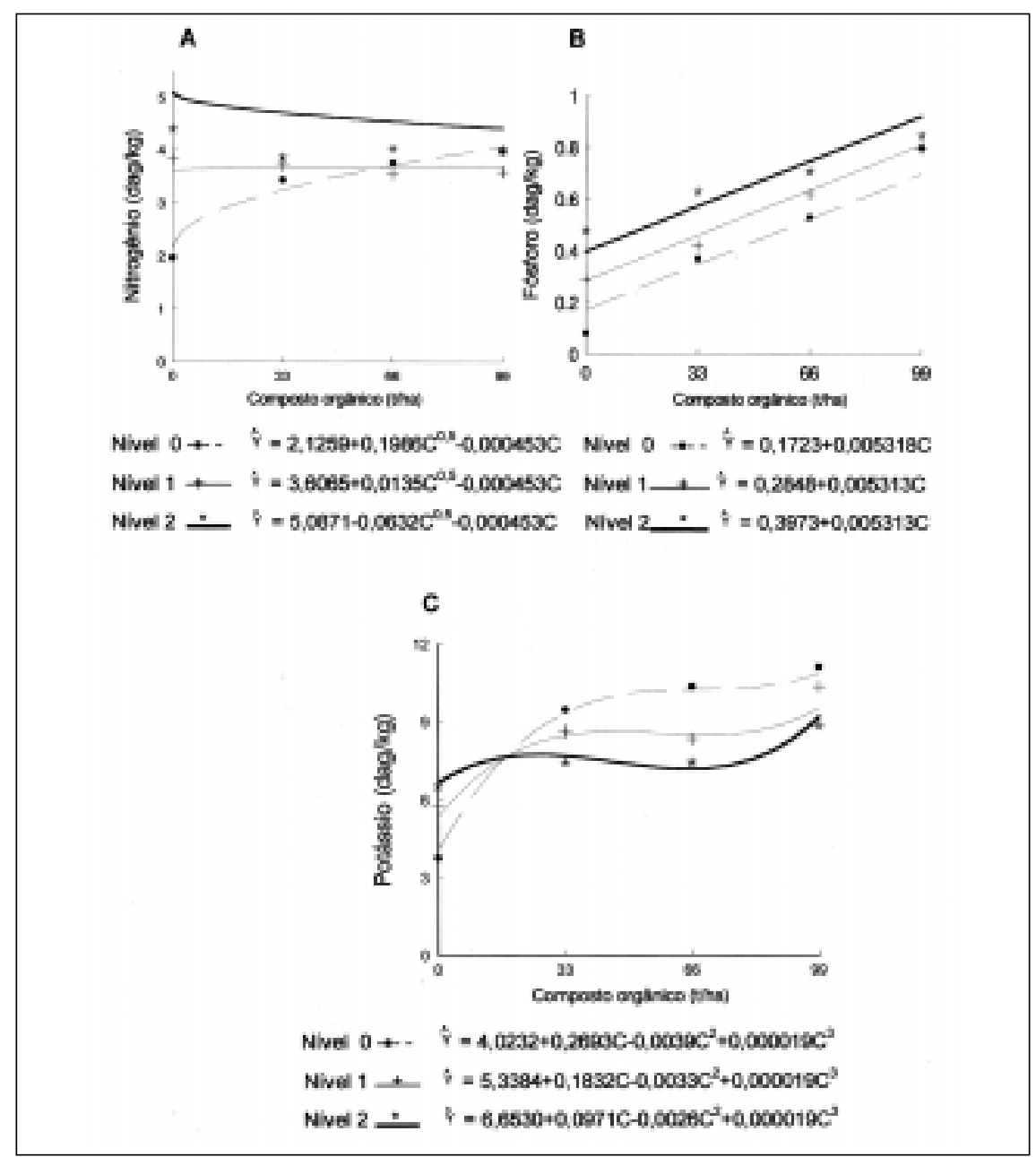

Figura 2. Concentração de nitrogênio (A), fósforo (B) e potássio (C) em folhas e alface, em função das doses de composto orgânico e dos níveis de adubo mineral. Viçosa, UFV, 1988. 
tal foliar (Figura 2B). As concentrações estimadas nas doses que promoveram as produtividades máximas foram de 0,41 ; 0,44 e $0,61 \%$, para os níveis 0,1 e 2 de adubos minerais, respectivamente. $\mathrm{O}$ uso exclusivo do composto (nível 0) parece ter sido suficiente para atender a exigência de fósforo pela planta, porque segundo Garcia et al. (1982), os teores detectados caracterizam plantas de alface adequadamente supridas com $\mathrm{P}$.

As doses crescentes do composto orgânico provocaram elevações nos teores foliares de potássio nos três níveis de adubação mineral (Figura 2C). As equações de regressão ajustadas demonstram que os teores de sódio aumentaram e os de cálcio diminuíram (Tabela 1). Esses resultados dão indícios de que os maiores incrementos de $\mathrm{K}$ e de $\mathrm{Na}$ dificultaram a absorção de $\mathrm{Ca}$, pois sabe-se que o Ca pode ser substituído por cátions monovalentes quando esses estão mais disponíveis nos sítios de absorção radiculares (Marschner, 1986, Mengel \& Kirkby, 1987). Estima-se que 2/3 do K de adubos orgânicos incorporados ao solo são prontamente solúveis em água e requerem apenas transformações físicas para serem liberados à solução do solo (Siqueira, 1988).

Várias fontes orgânicas veiculam teores consideráveis de $\mathrm{K}$ e $\mathrm{Na}$ ao solo, em liberações que não dependem da velocidade de mineralização da matéria orgânica (Siqueira, 1988). Isso pode possibilitar que toda a necessidade das plantas seja suprida por fontes orgânicas. Neste trabalho, as altas doses de matéria orgânica adicionadas ao solo promoveram grandes acúmulos nas folhas desses dois nutrientes (Tabela $1 \mathrm{e}$ Figura 2C), podendo ter ocorrido "consumo de luxo" e mesmo efeitos fitotóxicos. O problema da alta disponibilidade de $\mathrm{K}$ e Na reside na competição com Ca por absorção e no aumento da salinidade do solo (Marschner, 1986; Mengel \& Kirkby, 1987).

As doses de adubos orgânicos que maximizam a produtividade e diminuem custos, complementadas com fontes minerais solúveis, poderiam ser estabelecidas para condições regionais, visando a melhoria de propriedades físicas e biológicas do solo e reduções de custos com adubação.

\section{LITERATURA CITADA}

ALISSON, F.E. Soil organic matter and its role in crop production. Amsterdan, Elsevier Scientific, 1973. 637 p. (Developments in soil Science, 3).

ASANO, J. Effect of organic manures on quality of vegetables. Japanese Agriculture Research Quality, v. 18, n. 1, p. 31-36, 1984.

CASSOL, P.C.; BECEGATTO, V.; ERNANI, P.R.; TIMMERMANN, C.; GIANELLO, C. Liberação de nitrogênio e potássio de estrume aplicado no solo. Anais do XXVI Congresso Brasileiro de Ciência do Solo. Rio de Janeiro, 1997. (Resumo n. 4-251, CD-ROM).

CHAE, Y.M.; TABATABAI, M.A. Mineralization of nitrogen in soils ammended with organic wastes. Journal of Environmental Quality, v. 15, n. 2, p. 193-198, 1986.

COMISSÃO DE FERTILIDADE DO SOLO DO ESTADO DE MINAS GERAIS Recomendações para o uso de corretivos e fertilizantes em Minas Gerais. 4a Aproximação. Lavras, MG, 1989. $176 \mathrm{p}$.

DEFELIPO, B.V.; RIBEIRO, A.C. Análise química do solo (Metodologia). Viçosa, MG, UFV, Impr. Univ., 1981. 17 p. (Boletim de Extensão, 29).

GARCIA, L.L.C.; HAAG, H.P.; NETO, V.D. Nutrição mineral de hortaliças. Deficiências de macronutrientes em alface (Lactuca sativa L.), cv. Brasil 48 e Clause's Aurélia. Anais da E.S.A. Luiz de Queiroz, Piracicaba, v. 39, p. 349-362, 1982.

GIANELLO, C.; ERNANI, P.R. Rendimento da matéria seca de milho e alterações na composição química do solo pela incorporação de quantidades crescentes de cama de frangos, em casa de vegetação. Revista Brasileira de Ciência do Solo, Campinas, v. 7, p. 285-290. 1983.

GROHMANN, F. Superfície específica do solo de unidades de mapeamento do Estado de São Paulo. II. Influência da matéria orgânica, dos óxidos de Fe e dos cátions trocáveis, na superfície específica total do solo. Bragantia, Campinas, v. 31, n. 14. p. 167-185, 1972.

HARTRATH, H. Manuring of butterhead lettuce. Horticultural Abstract, v. 56, p. 7739, 1986.

HAWORTH, F.; CLEAVER, T.J. The effects of different treatments on the yield and mineral composition of winter lettuce. Journal of Horticultural Science, v. 42,: p. 23-29, 1967.

JACKSON, M.L. Soil chemical analysis. New Jersey, Prentice Hall, 1958. 498 p.

KIEHL, E.J. Fertilizantes orgânicos. $1^{a}$ ed., São Paulo, Ed. Agron. Ceres, 1985. 492 p.
LOURES, E.G. Produção de composto no meio rural. Viçosa, UFV, Imprensa Universitária, 1983. 5 p. (Informe Técnico, 17).

MARSCHNER, H. Mineral nutrition of higher plants. London, Academic Press Inc., 1986. $674 \mathrm{p}$.

MAZUR, N; VELLOSO, A.C.X.; SANTOS, G.A. Efeito do composto de resíduo urbano no $\mathrm{pH}$ e alumínio trocável em solo ácido. Revista brasileira de Ciência do Solo, Campinas, v. 7, p. 157-159, 1983.

MELO, F.A.F.; BRASIL SOBRINHO, M.O.C.; ARZOLLA, S.; SILVEIRA, R.I.; COBRA NETO, A. ; KIEHL, E.J. Fertilidade do solo. Piracicaba, Nobel, 1984. 400 p.

MENGEL, K.; KIRKBY, E.A. Principles of plant nutrition. Bern, International Potash Institute, 1987. 687 p.

NOVAIS, R.F.; NEVES, J.C.L.; BARROS, N.F. Ensaio em ambiente controlado. In: OLIVEIRA, A.J. de; GARRIDO, W.E.; ARAÚJO, J.D.; LOURENÇO, S. (Coord). Métodos de pesquisa em fertilidade do solo. Brasília, EMBRAPA, 1991. p.189-253.

RODRIGUES, E.T. Efeitos das adubações orgânica e mineral sobre o acúmulo de nutrientes e sobre o crescimento da alface (Lactuca sativa L). Viçosa, MG, UFV, 1990. 60 p. (Tese mestrado).

SARRUGE, J.R.; HAAG, H.P. Análises químicas em plantas. Piracicaba, E.S.A. "Luiz de Queiroz”, USP, 1974. 56 p.

SHORTALL, J.G.; LIEBHARDT, C.W. Yield and growth of corn as affected bu poultry manure. Journal of Environmental Quality, v. 4, n. 2, p. 186-191, 1975.

SIQUEIRA, J.O. Biotecnologia do solo: fundamentos e perspectivas. Lavras, MEC/ABEAS, 1988. 235 p.

SONNENBERG, P.E. Olericultura especial. Primeira Parte. $5^{a}$ ed., Goiânia, 1985. 189 p.

TROCMÉ, S.; BRIANT, A. Organic and nitrogenous manuring in intensive market gardening. Horticultural abstract, v. 33, n. 1, p. 57,1963. (resumo n. 777).

VALENTE, C.F. Efeito do biofertilizante, em diferentes níveis de adubação química, sobre o solo e sobre a produção do feijão cultivado em casa de vegetação. Viçosa, UFV, Impr. Univ., 1985. 48 p. (Tese mestrado.).

WANG, S.H.; LOHR, V.I.; COFFEY, D.L. Growth response of selected vegetabel crops to spent mushroom compost application in a controlled environment. Plant and Soil, v. 82, n. 1, p. 3140, 1984.

ZINK, F.W.; YAMAGUCHI, M. Studies on the growth rate and nutrient absorption of head lettuce. Hilgardia, v. 32, n. 11, p. 471-500, 1962. 